\title{
Factores relacionados con la empleabilidad de egresados de la carrera universitaria de gastronomía en Lima, Perú
}

\author{
Employability related factors of gastronomy university \\ school graduates in Lima, Peru
}

\author{
(iD) Yrene C. Uribe Hernández', $\unrhd$ (iDEsteban V. Horna Bances², (iD Stalein J. Tamara Tamariz ${ }^{3}$ \\ (iD) Carmen P. Tello Aguilar ${ }^{4}$ y iD Gloria M. Torres Molina ${ }^{5}$
}

\author{
1 Universidad Nacional de Cañete. Lima, Perú \\ ${ }^{2}$ Universidad Le Cordon Bleu. Lima, Perú \\ ${ }^{3}$ Universidad Privada del Norte. Lima, Perú \\ ${ }^{4}$ Universidad César Vallejo. Lima, Perú \\ ${ }^{5}$ Servicio Nacional de Adiestramiento en Trabajo Industrial - SENATI. Lima, Perú
}

\begin{tabular}{llll}
\hline Recibido: 22/01/2018 & Revisado: 01/03/2019 & Aceptado: 12/05/2019 & Publicado: 30/06/2019 \\
\hline
\end{tabular}

\section{RESUMEN}

La investigación tuvo como objetivo Identificar los principales indicadores de percepción sobre seguridad ciudadana en el distrito del Magdalena del Mar. Se encuestaron 224 personas de 18 años hacia adelante. Se realizó una estadística descriptiva con intervalos de confianza para estimar las proporciones poblacionales de la variable en estudio "percepción". Como conclusión relevante fue que, se identificó que la principal causa de la inseguridad ciudadana en el distrito de Magdalena del Mar son los robos.

Los habitantes piden más organización social para sentirse más seguros en el distrito. Los habitantes califican como regular la eficiencia de los policías ante la presencia de diferentes actos de seguridad ciudadana. Más de la mitad de los encuestados piensa que la situación sobre la seguridad ciudadana en el distrito de Magdalena del Mar en los últimos doce meses no ha tenido cambios significativos. Más de un tercio de los habitantes piensa que el principal motivo por el cual se produce la inseguridad ciudadana es la falta de policías.

Menos del 50 \% de los habitantes del distrito piensa que el ejercicio que realiza la policía y serenazgo es eficiente. Referente a la percepción sobre la seguridad ciudadana en el distrito de Magdalena del Mar, la gran mayoría de las personas encuestadas sienten la falta de seguridad por parte de las autoridades y que; en lugar de ir mejorando, va en aumento.

Palabras clave: Seguridad ciudadana, asaltos, normas viales.

\section{ABSTRACT}

The objective of the research was to identify the main perception indicators on citizen security in the Magdalena del Mar district. 224 people 18 years of age and older were surveyed. A descriptive statistic was carried out with confidence intervals to estimate the population proportions of the variable under study "perception". As a relevant conclusion, it was identified that the main cause of citizen insecurity in the Magdalena del Mar district is robbery.

The inhabitants ask for more social organization to feel more secure in the district. The inhabitants qualify as regulating the efficiency of the police in the presence of different acts of citizen security. More than half of the respondents think that the situation on citizen security in 
the Magdalena del Mar district in the last twelve months has not had significant changes.

More than a third of the inhabitants think that the main reason for citizen insecurity is the lack of police. Less than $50 \%$ of the inhabitants of the district think that the exercise carried out by the police and municipal security agents is efficient.

Regarding the perception of citizen security in the Magdalena del Mar district, the vast majority of the people surveyed feel the lack of security by the authorities and that instead of improving, it is increasing.

Keywords: Citizen security, assaults, road regulations.

\section{INTRODUCCIÓN}

La gastronomía es una actividad que, como profesión, es de relativamente reciente creación. Nace en el Perú con la creación de la universidad Le Cordon Bleu (ULCB), bajo la forma de sociedad anónima cerrada, fundada y promovida bajo la administración del Consejo Nacional para la Autorización y funcionamiento de Universidades (CONAFU) con fecha 04 de mayo de 2009 y resolución $\mathrm{N}^{\circ}$ 220-2009-CONAFU, para brindar servicios educativos de nivel universitario, mediante las carreras profesionales de administración de negocios turísticos y hoteleros; gastronomía y arte culinario; industrias alimentarias; y nutrición, salud y técnicas alimentarias.

Uno de los aspectos importantes a desarrollar son las investigaciones para saber las características que influyen en el desarrollo de las carreras en gastronomía, dentro de esto es indudable la creciente importancia que está cobrando la empleabilidad, característica que nos permite dirigir la mejora de los planes de estudios con la finalidad de que la ULCB mejore su oferta profesional.

En Valencia, España, se ha realizado una investigación para, por una parte, contrastar si mediante un mecanismo de reducción de la dimensión es posible sintetizar las competencias en los siete conjuntos que plantea el Libro Verde de empleabilidad de los titulados universitarios de la comunidad valenciana; $y$, por otra parte, analizar el impacto de dichas competencias y de otras variables sociodemográficas y relacionadas con los estudios en el desempleo (MartínGonzález y Pérez, 2015).

Por otro lado, en la universidad de Oviedo, se desarrolló un estudio para conocer la empleabilidad y la mejora laboral que obtienen actualmente los egresados del máster profesionalizante en formación del profesorado de educación secundaria obligatoria, bachillerato y formación profesional.

Se analizaron las tendencias relacionadas con la ocupación, sueldo y tiempo que tardan en colocarse en el mercado laboral, así como la satisfacción con la formación recibida y la aplicación de los conocimientos adquiridos en el empleo obtenido (Pascual y Díaz, 2016).

También, se ha estudiado la empleabilidad de los estudiantes de las carreras de enfermería y psicología respecto a la búsqueda, obtención y expectativas de un trabajo en la universidad Autónoma del Estado de Morelos, Cuernavaca, México 
(Paz-Rodríguez, Betanzos-Díaz y UribeBarrera, 2014).

Además, en Ecuador se ha caracterizado la situación actual de la empleabilidad de graduados universitarios en el contexto latinoamericano, ecuatoriano y en la carrera de administración de empresas de la universidad regional Autónoma de los Andes (UNIANDES) (Álvarez y Romero, 2015).

También, se ha estudiado al docente en España para conocer las demandas de formación del mercado laboral en términos de competencias para el aprendizaje a lo largo de la vida (LLL) a través de la importancia que egresados y empleadores les conceden y traducirlas en recomendaciones y propuestas de formación para el profesorado universitario (Jato, Cajidel, Muñoz y García, 2016).

La ULCB ha estado realizando últimamente estudios que le permita tener una visión más cercana de la percepción que tienen los actores de su accionar. Es así como se encuestó a representantes de 125 empresas vinculadas con las carreras profesionales que la ULCB oferta (Uribe et al, 2018).

La ULCB está enclavada en el distrito de Magdalena y Miraflores; considerado este último como el principal distrito turístico del Perú, y Magdalena del Mar como un distrito de grandes posibilidades de desarrollo.

Ambos poseen una amplia gama de empresas de servicios gastronómicos con los que están vinculados las carreras que oferta la ULCB.
Por estas razones, se ha realizado el presente estudio con el objetivo de determinar los factores relacionados con la empleabilidad de los egresados de la carrera universitaria de gastronomía ofertada por la universidad Le Cordon Bleu en Lima, Perú. Los resultados serán de suma utilidad para que la ULCB cumpla con sus funciones, y se convierta en la institución proveedora de soluciones científicas y tecnológicas a problemas gastronómicos y de profesionales adecuados a las necesidades de su zona de influencia y del país.

\section{MATERIALES Y MÉTODOS}

Tipo de estudio: El estudio fue correlacional, transversal y cuantitativo.

Muestra: La muestra se determinó al azar, de acuerdo con los datos poblacionales actualizados de la población o universo, en el caso de estudiantes y profesores de la universidad Le Cordon Bleu, a los que les correspondió una muestra de 114 estudiantes y 27 docentes respectivamente, y en el caso de egresados y empleadores una muestra irrestricta, pues la información se colectó de los que desearon participar en la encuesta.

Recolección de la información: Los datos se tomaron haciendo uso de encuestas en forma presencial para el caso de los profesores y estudiantes, y de encuestas virtuales para el caso de los egresados y empleadores.

Técnica y procesamiento de la información: La técnica para procesar la información fue mediante la estadística inferencial haciéndose usó del modelo de regresión logística. Se usó el software Excel para los análisis. 
RESULTADOS

Percepción de los estudiantes sobre factores y capacidades relacionadas con su empleabilidad.

Se encuestaron 114 estudiantes de los dos últimos ciclos de la carrera de gastronomía y gestión empresarial, de ellos el $43 \%$ pertenecían al género femenino y $57 \%$ al masculino.

En líneas generales los mayores puntajes obtenidos bajo la percepción de los estudiantes fueron: casi siempre realizan una actividad con buena disposición y siempre el creer que serpia un buen profesional si logra obtener un trabajo en su carrera.

Por otro lado, los factores individuales que influyen en la empleabilidad fueron siempre aceptas tu responsabilidad al cometer un error, le gusta mantener la armonía entre sus compañeros; en menor grado, acepta la crítica por parte de sus compañeros (fig. 1).

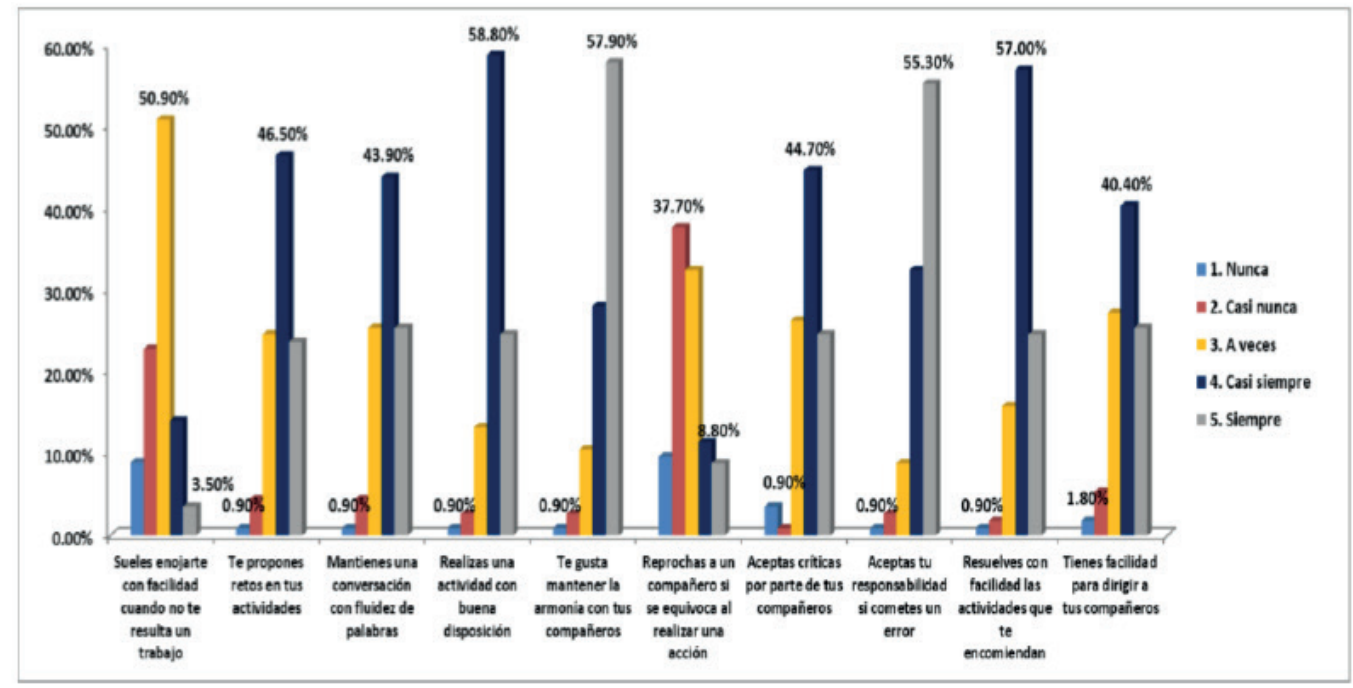

Figura 1. Factores individuales que influyen en la empleabilidad.

Las capacidades de adaptación con facilidad a los nuevos cambios tecnológicos y de aprender rápidamente una tarea encomendada siempre tuvieron la mayor incidencia para obtener empleo; y para casi siempre fueron tener buena disposición y tomar las decisiones deforma acertada y ágil, saber negociar y resolver conflictos y saber maximizar los recursos que les brindan (fig. 2). 


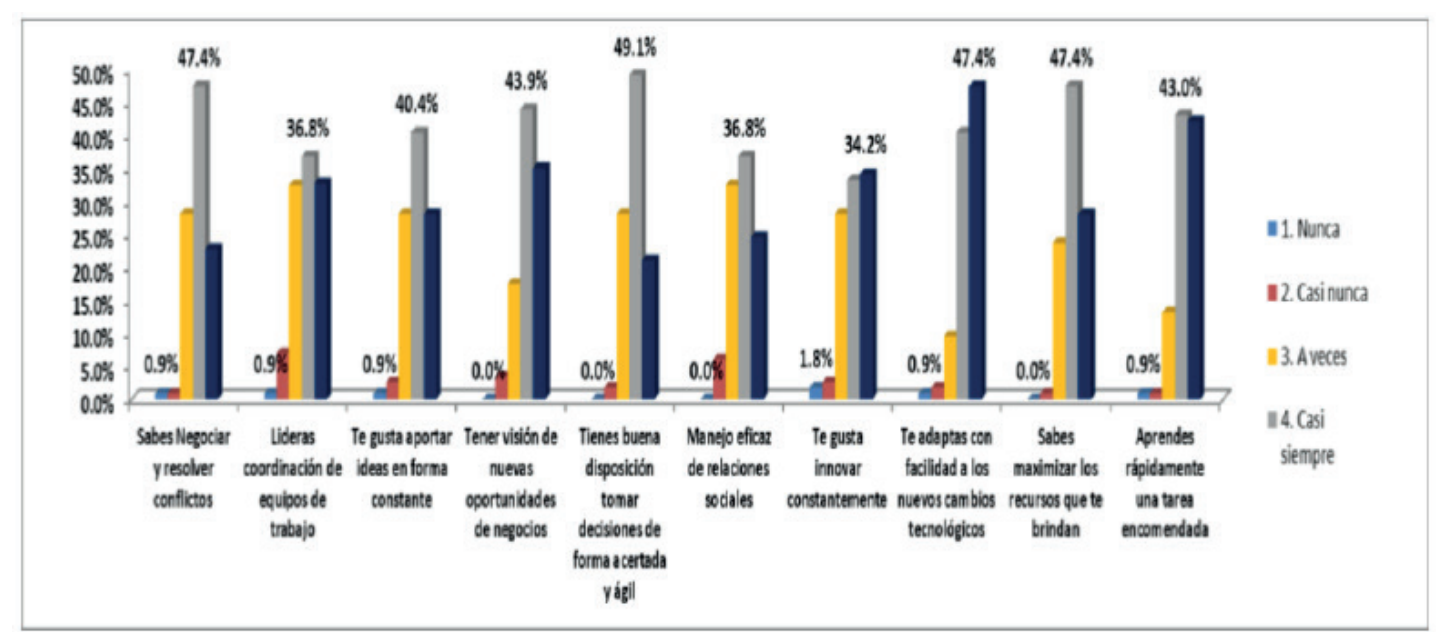

Figura 2. Capacidades para obtener un empleo.

De la misma manera, cuando se estudiaron las capacidades para mantener el empleo, las que tuvieron mayor valor al registrarse como siempre fueron el tener respeto por la naturaleza y el tener respeto por el orden público y privado; y como casi siempre tener una rápida recuperación ante la frustración por cualquier falla o error (fig. 3).

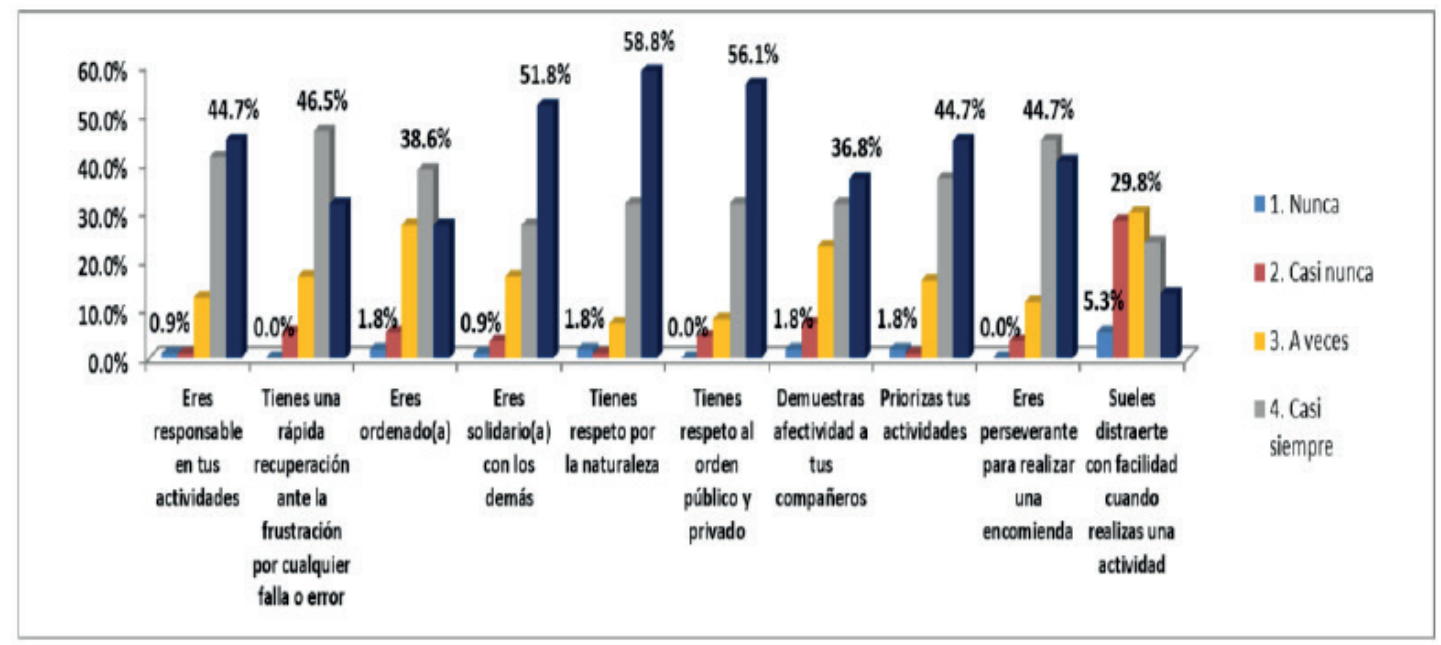

Figura 3. Capacidades para mantener un empleo. 
También se buscó establecer la significación estadística entre las relaciones que pueden establecerse entre la percepción de empleabilidad y diferentes capacidades tales como la de obtener empleo, la de mantener empleo, la opinión sobre lo que se piensa que es calidad de un puesto de trabajo, así como percepción sobre capacidades de desempeño laboral y factores individuales para conseguir empleo, las actitudes para buscar empleo y los factores que influyen en un contrato laboral, el resultado fue que todas las relaciones mencionadas fueron significativamente estadísticas al obtener valores por debajo de 0,05 (tabla 1).

Tabla 1. Relaciones de percepción de la empleabilidad y diferentes capacidades.

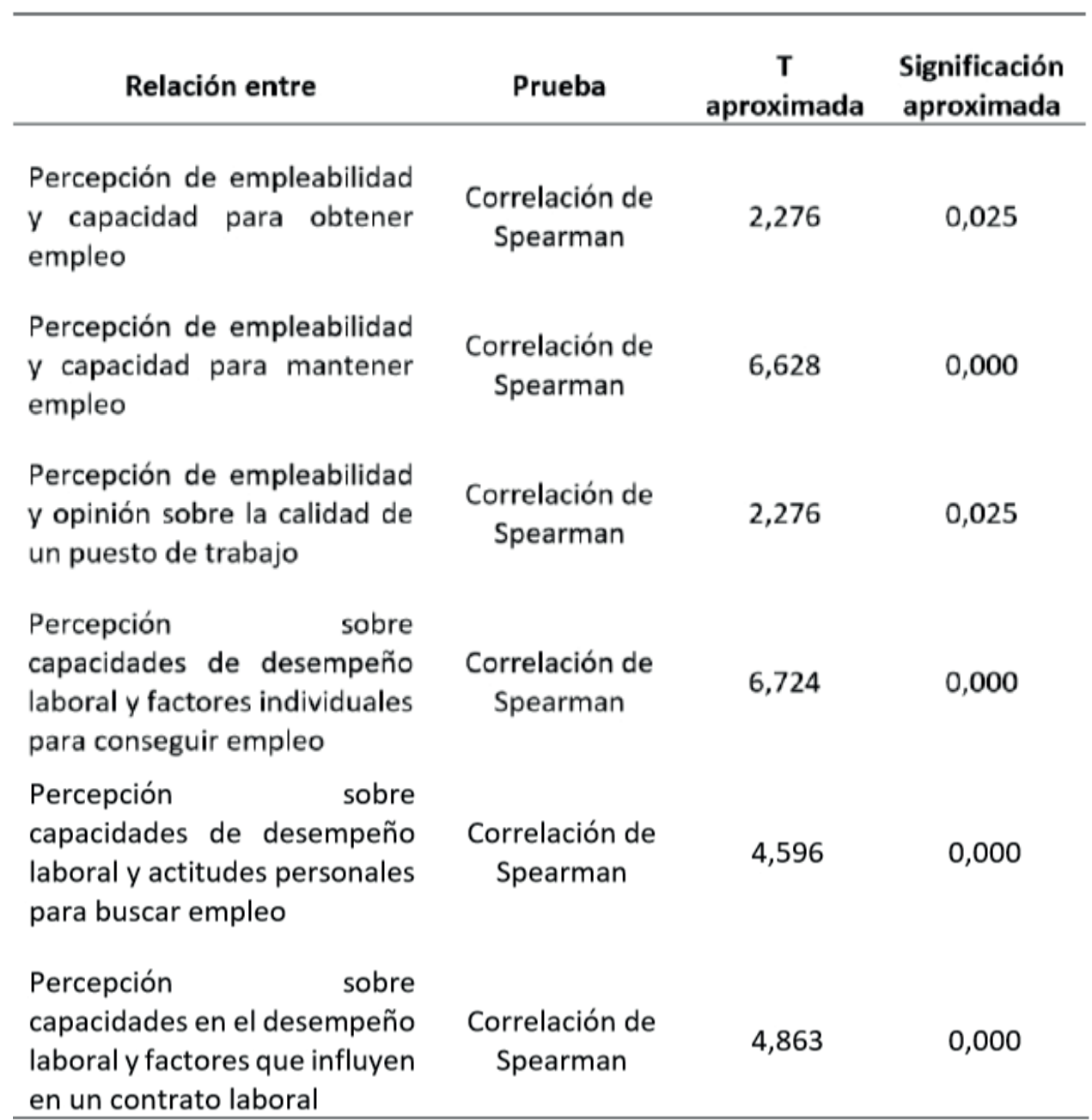

$\begin{array}{lcrl}\begin{array}{l}\text { Percepción de los egresados sobre } \\ \text { capacidades consideraron influyentes para acceder a un }\end{array} \\ \text { empleabilidad } & \text { adquiridas } & \text { para } & \begin{array}{l}\text { contrato laboral fueron en mayor medida el } \\ \text { estado civil, los contactos que se puedan }\end{array} \\ \text { Por otrolado, los factores quelos egresados } & \text { tener y la experiencia laboral (fig. 4) }\end{array}$




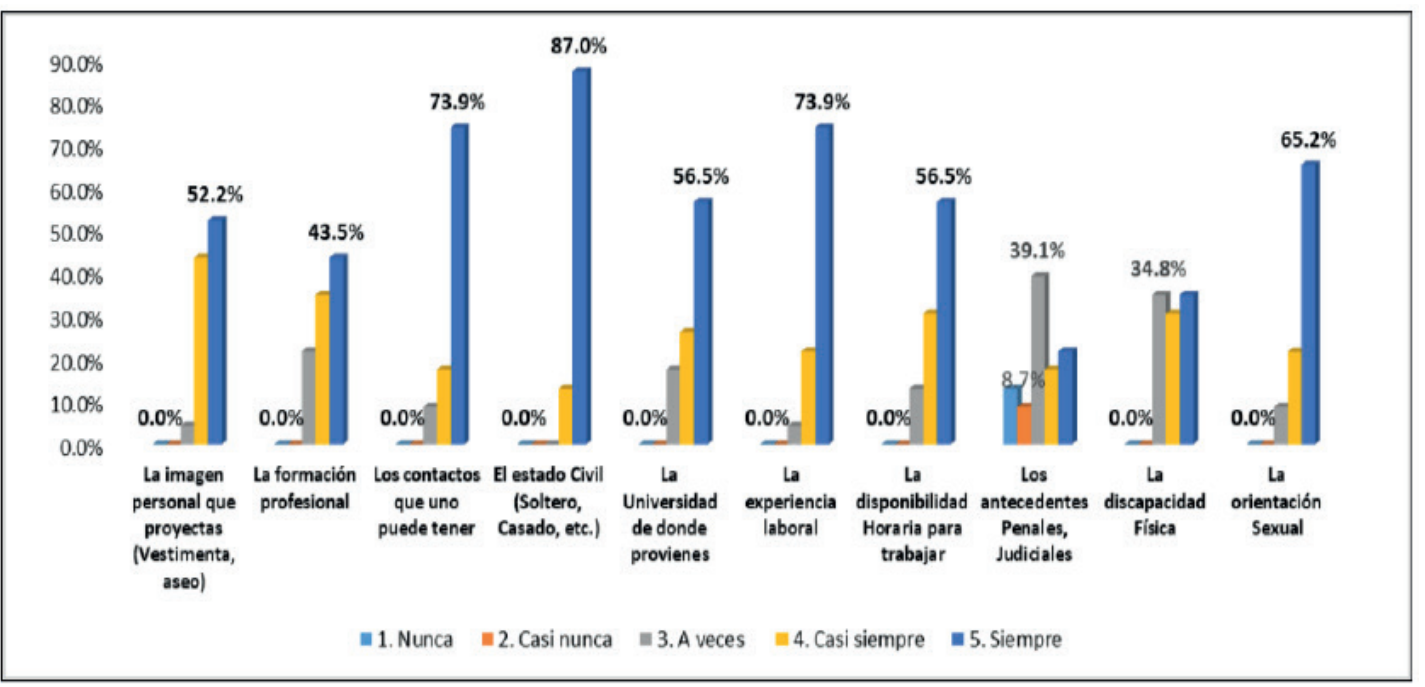

Figura 4. Factores externos que influyen en el acceso a un contrato laboral.

La principal capacidad que se consideró siempre para obtener empleo fue el saber negociar y resolver conflictos, seguido por aprender rápidamente una tarea encomendada; para casi siempre, fue el saber maximizar los recursos que les brindan y tener buena visión de nuevas oportunidades de negocios (fig. 5).

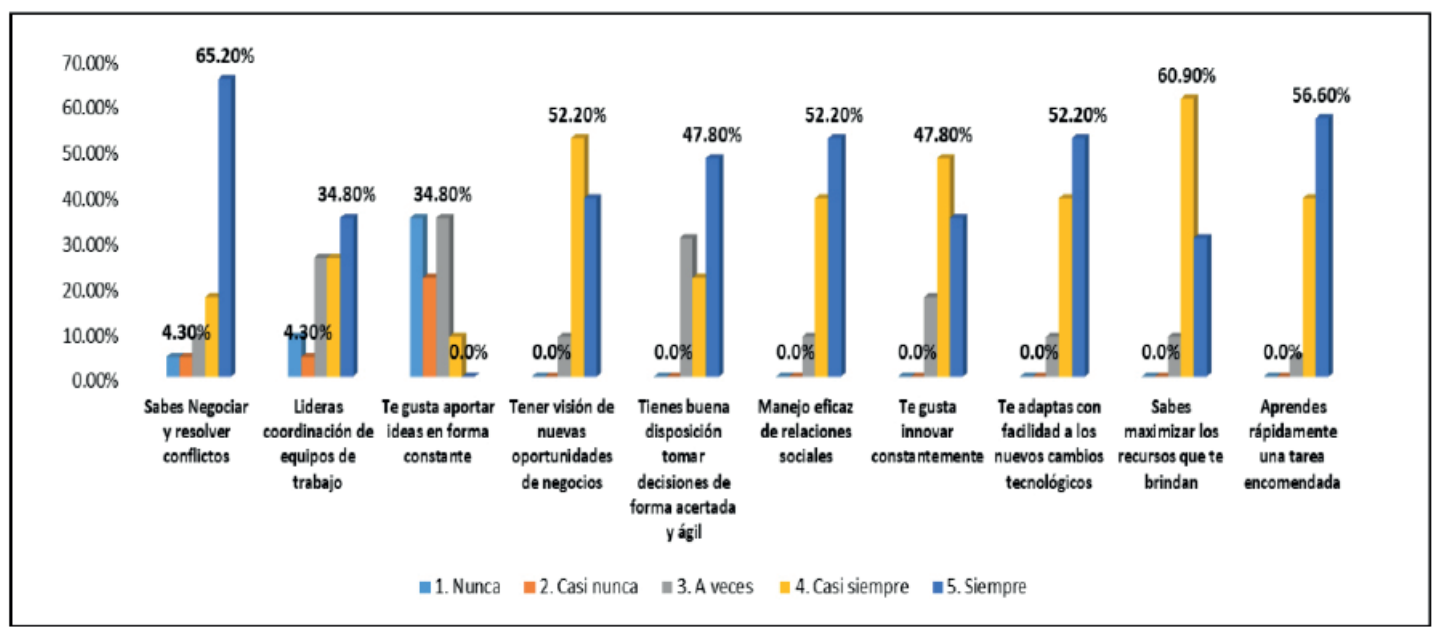

Figura 5. Capacidades para obtener un empleo.

Cuando de mantener el empleo se trató, las principales capacidades que se consideraron siempre como más importantes fueron demostrar afectividad a sus compañeros, tener respeto al orden público y privado y ser perseverante; para el caso de casi siempre fueron ser responsable en sus actividades, tener una rápida recuperación ante la frustración por cualquier falla o error ser ordenado. Para el factor negativo de soler distraerse con facilidad el mayor valor fue para a veces (fig. 6). 


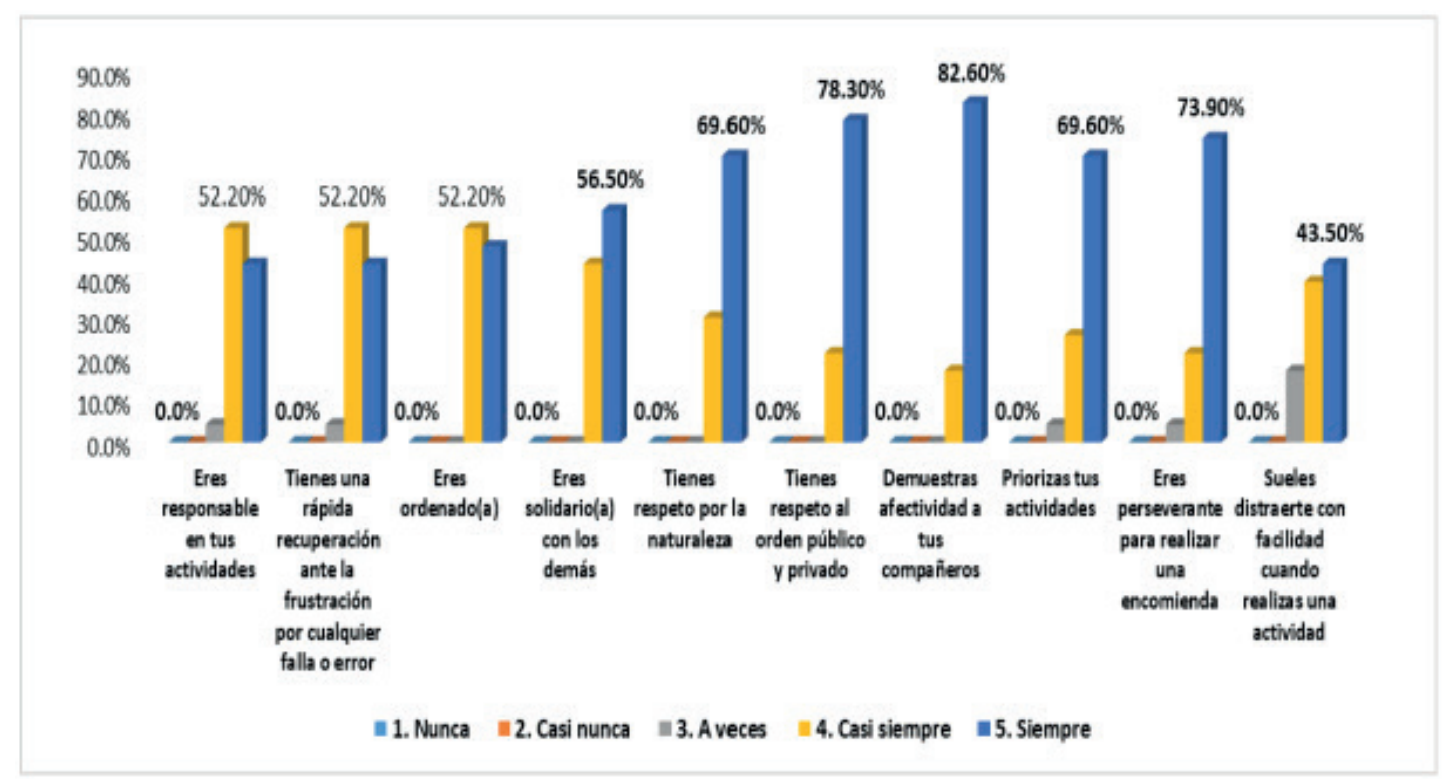

Figura 6. Capacidades para mantener un empleo.

Los factores individuales y las actitudes personales de los egresados incidieron significativamente en la capacidad de mantener un empleo. Según la tabla 1 de resumen de modelos, el 82,4 \% de la variación en la capacidad de mantener el empleo es explicada por las actitudes personales y los factores individuales; la posibilidad de que la capacidad de mantener un empleo sea buena es mayor 19,842 veces si los factores individuales fueran buenos y 40,298 veces si las actitudes personales disminuyeran.

Cuando se evaluó el nivel de satisfacción con los servicios prestados por la ULCB, la mejor respuesta fue para el nivel de la formación recibida, seguida por el sistema de seguimiento del egresado, donde consideraron que era mayormente bueno (fig. 7).

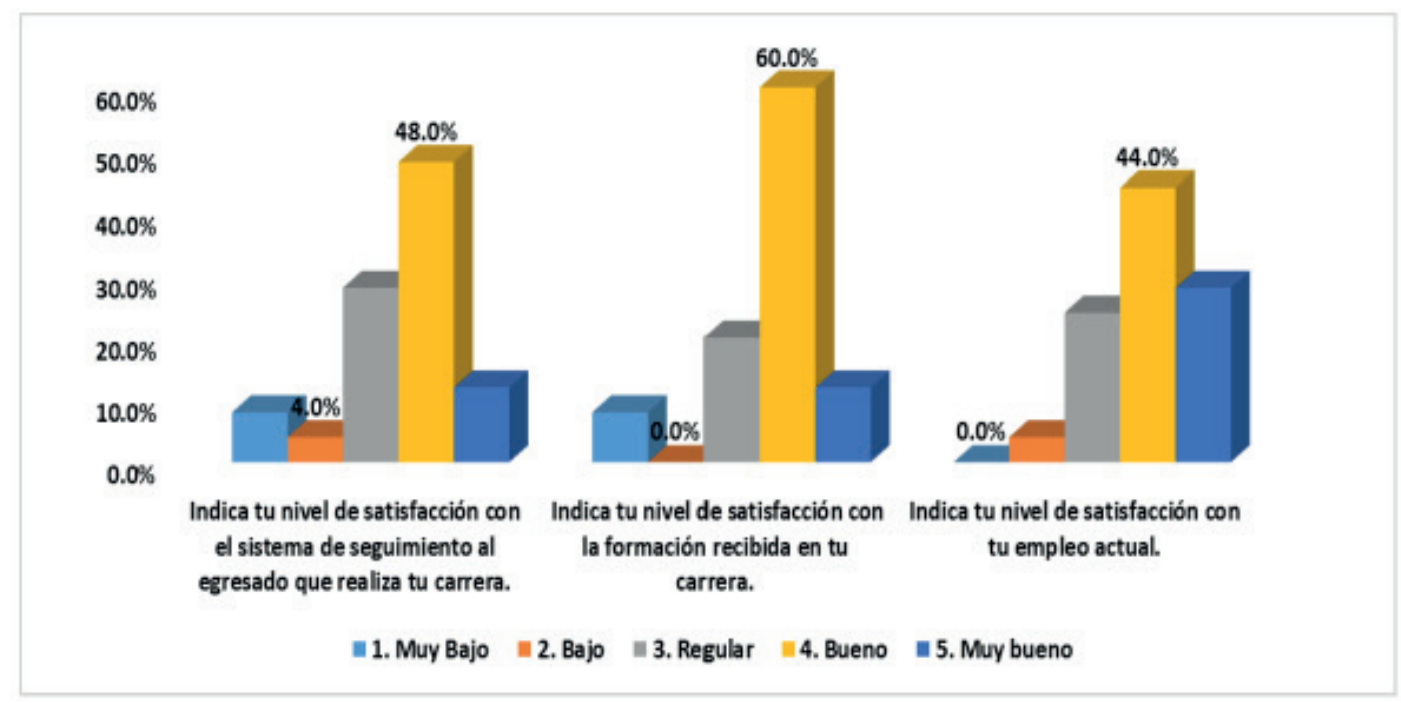

Figura 7. Nivel de satisfacción con servicios académicos de la ULCB. 
Percepción de los docentes sobre y en segundo lugar varias como tener perfil capacidades adquiridas para la de líder, ser solidario con sus compañeros, empleabilidad mostrarmanejo deemociones ante situaciones Se midió el grado de satisfacción de los complicadas. Cuando se acumulan los valores docentes con las capacidades adquiridas por muy bueno y bueno las capacidades relevantes los estudiantes que les permita conseguir fueron el ser solidario con sus compañeros de trabajo. Los resultados muestran que la trabajo, el mostrar manejo de sus emociones capacidad que tuvo un mayor registro como ante situaciones complicadas y el mostrar bueno fue el mostrar respeto por la autoridad respeto por el ambiente (tabla 2).

Tabla 2. Grado de satisfacción con capacidades obtenidas para conseguir trabajo.

\begin{tabular}{lccccc}
\hline Capacidad & Muy Bajo & Bajo & Regular & Bueno & Muy bueno \\
\hline Capacidad para resolver problemas & 11,1 & 7,4 & 33,3 & 25,9 & 22,2 \\
Innovación y creatividad & 3,7 & 14,8 & 25,9 & 33,3 & 22,2 \\
Manejo de herramientas de trabajo & 11,1 & 14,8 & 14,8 & 40,7 & 18,5 \\
manual (cocina) & & & & & \\
Puntualidad & 7,4 & 18,5 & 29,6 & 29,6 & 14,8 \\
Nivel de responsabilidad en actividades & 11,1 & 11,1 & 22,2 & 40,7 & 14,8 \\
encomendadas & & & & & \\
Tiene perfil de líder & 3,7 & 3,7 & 44,4 & 44,4 & 3,7 \\
Elabora y redacta informes de acuerdo & 3,7 & 14,8 & 44,4 & 29,6 & 7,4 \\
con criterios prestablecidos & & & & & \\
Posee conocimientos científicos & 11,1 & 11,1 & 40,7 & 33,3 & 3,7 \\
Muestra respeto por la autoridad & 7,4 & 11,1 & 29,6 & 25,9 & 25,9 \\
Es solidario con sus compañeros de & 3,7 & 14,8 & 14,8 & 44,4 & 22,2 \\
trabajo & & & & & 18,5 \\
Muestra manejo de sus emociones ante & 7,4 & 11,1 & 18,5 & 44,4 & \\
situaciones complicadas & & & & & 7,4 \\
Su desempeño le inspira confianza & 18,5 & 7,4 & 25,9 & 40,7 & \\
\hline
\end{tabular}




\section{Percepción de empleadores}

Los empleadores que respondieron fueron 13 y en un $61,5 \%$ pertenecían al género femenino, correspondiéndole el 38,5 \% restante al masculino. Otra diferencia fue el que se desempeñaban en instituciones privadas en un $92,3 \%$ y solo un $7,7 \%$ en institución pública.

Cuando se estudió su grado de satisfacción por el desempeño del egresado de la ULCB, se obtuvo la tabla 3, nos muestra que, los porcentajes más bajos dentro del rango positivo, que comprendería a bueno, muy bueno y excelente se adjudicaron a la categoría de excelente; por otro lado, los más altos porcentajes fueron obtenidos en la calificación de muy bueno para la característica de ser ordenado con sus actividades, y su desenvolvimiento durante presentaciones de trabajos encomendados; en el caso de la calificación de bueno, los más altos porcentajes se atribuyeron a la capacidad para resolver problemas y la posesión de conocimientos científicos.

Tabla 3. Percepción de los empleadores

\begin{tabular}{lcccc}
\hline Característica & Bajo & Regular & Bueno & Excelente \\
\hline Capacidad para resolver problemas & 69,2 & 30,8 & \\
Cualidades de innovación y creatividad & 53,8 & 30,8 & 15,4 \\
Manejo de herramientas de trabajo manual & 7,7 & 30,8 & 46,2 & 15,4 \\
(cocina) & & & \\
& & 15,4 & 46,2 & 38,5 \\
Puntualidad & & & \\
Nivel de responsabilidad en actividades & 30,8 & 53,8 & 15,4 \\
encomendadas & & & \\
Nivel de iniciativa ante situaciones nuevas & & 30,8 & 46,2 & 23,1 \\
Escribe y habla idiomas extranjeros o lenguas & 7,7 & 23,1 & 53,8 & 15,4 \\
nativas & & & & \\
Tiene perfil de líder & 15,4 & 38,5 & 46,2 & \\
Muestra respeto por la autoridad & 7,7 & 23,1 & 30,8 & 38,5 \\
Es solidario con sus compañeros de trabajo & & 15,4 & 46,1 & 38,5 \\
Muestra manejo de sus emociones ante & 7,7 & 30,8 & 53,8 & 7,7 \\
situaciones complicadas & & & & \\
Es ordenado en sus actividades & & 23,1 & 69,2 & 7,7 \\
Muestra compromiso con centro laboral & 23,1 & 53,8 & 23,1 \\
Muestra deseos de superación & 7,7 & 7,7 & 46,2 & 38,5 \\
Muestra conformismo & 23,1 & 15,4 & 7,7 \\
\hline
\end{tabular}




\section{Necesidad de capacitación del egresado:}

El $61,5 \%$ de empleadores consideraron que nuestros egresados sí tenían necesidad de capacitación y el 38,5 \% que no. Las recomendaciones que hicieron los que consideraronqueera necesarialacapacitación fueron: Trabajo en equipo, humildad, formalidad, cumplimiento de compromisos, comunicación asertiva, manejo de emociones y solución de problemas, más confianza en sí mismo y creatividad.

\section{DISCUSIÓN}

Nuestro trabajo ha cubierto varios aspectos relacionados con la empleabilidad de los egresados de la carrera de gastronomía de la ULCB.

Aun estaría pendiente el establecer sus porcentajes de empleabilidad, en qué tipo de trabajo se están desenvolviendo, entre otros. Si bien no se ha encontrado estudios similares para la carrera de gastronomía, se han realizado una serie de estudios en diferentes partes del mundo y estudiado diferentes carreras, como por ejemplo, el realizado en graduados de psicología en España para identificar los elementos que participan en la gestión de la carrera para el desarrollo de la empleabilidad, llegándose a las siguientes conclusiones:

Como puntos esenciales se debe: 1) es importante la generación de una red de contactos como posibilitadora de espacios de vinculación, así como el fomento de habilidades interpersonales, el involucramiento activo en la propia gestión de la carrera, la adaptación o habilidad para ajustarse fácilmente a los cambios y estar disponible constantemente, 2) debe potenciarse la capacidad de adaptación y el compromiso con la identidad, desarrollo y ética profesional, y 3) la autogestión de la carrera profesional debe darse desde la formación inicial (Llanes, Figuera y Torrado, 2017).

Es de igual manera importante conocer la situación de los egresados empleados, ante la posibilidad de que no se estén desempeñando en espacios propios de su carrera y el subempleo; si bien no hemos diseñado nuestra investigación en esos términos, debería considerarse una investigación posterior e incluso proponer acciones que permitan evitarlo.

Una evidencia importante es el estudio realizado por Muñoz (2006) en México sobrelos factores que intervienen en la determinación del desempleo de los egresados de educación superior y las políticas mediante las cuales se ha intentado evitarlo.

Se propusieron políticas alternas para abatir el subempleo estructural como: 1) modificar los currículos de estudios, 2) vincular su investigación con los programas educativos de una forma interdisciplinaria, y 3) vincularse más estrechamente con los diferentes actores educativos y sociales.

La ULCB, desde el 2014 ha estado realizando estudios que le permita tener una visión más cercana de la percepción que tienen los actores de su accionar, es así como se encuestó a representantes de 125 empresas vinculadas con las carreras profesionales que la ULCB oferta.

Los resultados más resaltantes fueron: 1) dentro de la formación profesional del empresario, la capacidad de innovación constante;2) dentro del desarrollo empresarial, la realización de análisis periódicos de su organización; y 3) dentro del factor de éxito, la capacidad tecnológica y mejores costos. 


\section{CONCLUSIONES}

- Bajo la percepción de los estudiantes a) el factor externo más influyente fue siempre los antecedentes penales y judiciales, b) la principal capacidad para obtener empleo fue siempre el adaptarse con facilidad a los nuevos cambios tecnológicos, c) la principal capacidad para mantener el empleo fue el respeto por la naturaleza.

- Bajo la percepción de los egresados a) el factor externo que más influye en la empleabilidad fue siempre el estado civil, b) la principal capacidad para obtener un empleo fue siempre el saber negociar y resolver conflictos, c) la principal capacidad para mantener un empleo fue el demostrar afectividad por sus compañeros.

- Bajo la percepción de los docentes fue mayormente bueno el respeto por el ambiente, el manejo de herramientas tecnológicas, tener perfil de líder, ser solidario y mostrar manejo de sus emociones ante situaciones complicadas.

- Bajo la percepción de los empleadores fue mayormente bueno el ser ordenado en sus actividades y el desenvolvimiento en las presentaciones de trabajos encomendados.

- En su mayoría, los empleadores consideraron que los egresados requerían capacitación y esta capacitación debería ser en habilidades blandas, comportamiento más eficiente en el área de trabajo, idiomas y capacidad de síntesis y análisis.

- Hubo relación estadísticamente significativa entre la percepción de empleabilidad y diferentes capacidades tales como la de obtener empleo, la de mantener empleo, la opinión sobre lo que se piensa que es calidad de un puesto de trabajo, así como la percepción sobre capacidades de desempeño laboral y factores individuales para conseguir empleo, las actitudes para buscar empleo y los factores que influyen en un contrato laboral.

\section{REVISIÓN BIBLIOGRÁFICA}

Álvarez, J., y Romero, A. (2015). La empleabilidad de graduados universitarios en el contexto latinoamericano. Realidad de UNIANDES, Ecuador. Atenas 4(32):0115. Recuperado de: http://www.redalyc.org/ articulo. 0 a? id=478047208001

Jato, E.; Cajide, J., Muñoz, M., y García, B. (2016). La formación del profesorado universitario en competencias lifelong learning a partir de las demandas de empleadores y egresados. Revista de Investigación Educativa, 34(1), 69-85. DOI: http://dx.doi. org/10.6018/rie.34.1.215341

Llanes. J., Figuera, P., y Torrado, M. (2017). Desarrollo de la empleabilidad y gestión personal de la carrera de graduados en Pedagogía Revista Española de Orientación y Psicopedagogía, 28(2): 46-60. Recuperado de: $\quad$ http://www.redalyc.org/articulo. oa?id=338253221003

Martín-González, M., Ondé D., y Pérez, C. (2015) El impacto de las competencias en la empleabilidad de los titulados universitarios de las universidades. Investigaciones de Economía de la Educación. 4(18):687-707.

Muñoz, C. (2006). Determinantes de la empleabilidad de los jóvenes universitarios y alternativas para promoverla. Papeles de población (49):75-89. CIAEP UAEM.

Pascual, M., Díaz, S., y Rodriguez, C. (2016). Empleabilidad y ocupación laboral de los egresados del Máster en Formación del 
profesorado de Educación Secundaria obligatoria, bachillerato y formación profesional. Enseñanza y Teaching, 34(1):161177. doi: http://dx.doi.org/10.14201/ et2016341161177.

Suárez L., B. (2016). Empleabilidad: análisis del concepto. Revista de Investigación en Educación 14(1): 67-84. Recuperado de: http://webs.uvigo.es/reined/

Uribe, Y., Tamara, S., y Horna E. (2018). Relación Universidad-Empresa en restaurantes de tres distritos de LimaPerú. En Chávez, C., y Garrido, C. (Coord.) La vinculación Universidad-Empresa para el desarrollo integral con impacto social (272288). México, México: UDUAL y REDUEALCUE. ISBN 978-607-8496-08-02. 